\title{
NECROSE DA EXTREMIDADE LIVRE DO PÊNIS COMO COMPLICAÇÃO DE
} ACROPOSTITE-FIMOSE EM TOURO

Rogério Elias Rabelo1; Valcinir Aloísio Scalla Vulcani²; Bruno Moraes Assis ${ }^{3}$; Larissa Caroline Cordeiro de Andrade ${ }^{4}$; Rhavilla Santos de Oliveira ${ }^{4}$.

${ }^{1}$ Professor Adjunto, Setor de Cirurgia de Grandes Animais, Universidade Federal de Goiás/CAJ, Jataí, Goiás, Brasil. (rabelovet@yahoo.com.br)

${ }^{2}$ Professor Adjunto, Setor de Anatomia Animal, Universidade Federal de Goiás/CAJ, Jataí, Goiás, Brasil.

${ }^{3}$ Mestrando do Programa de Pós Graduação em Ciência Animal da Escola de Medicina Veterinária e Zootecnia da Universidade Federal de Goiás, Goiânia, Goiás, Brasil.

${ }^{4}$ Acadêmicas do Curso de Medicina Veterinária da Universidade Federal de Goiás/CAJ, Jataí, Goiás, Brasil.

Recebido em: 08/09/2015 - Aprovado em: 14/11/2015 - Publicado em: 01/12/2015 DOI: http://dx.doi.org/10.18677/Enciclopedia_Biosfera_2015_200

Dentre as enfermidades que acometem a genitália externa de touros, a acropostitefimose merece destaque. Em alguns casos, pode-se diagnosticar severo comprometimento da mucosa do folheto prepucial interno o que pode inviabilizar a cirurgia. Dentre as complicações, quando se opta pelo ato operatório, o comprometimento da extremidade livre do pênis não tem sido apontado como sequela frequente. Este trabalho objetiva descrever os achados clínicos e histopatológicos, de um caso incomum de estenose e fibrose do folheto prepucial interno com consequente necrose da extremidade livre do pênis, como intercorrência após a cirurgia de acropostite-fimose. Durante o procedimento foram colhidos fragmentos das áreas lesionadas para exame histopatológico. Não foi possível efetuar a correção cirúrgica da fimose prepucial, sendo a amputação de um segmento peniano necessária. O procedimento foi respaldado pelos achados histopatológicos, confirmando a gravidade do caso. O laudo histopatológico revelou glande com áreas focalmente extensas e superficiais de necrose, dentre outros achados.

PALAVRAS-CHAVE: Bovinos, genitália externa, impotência coeundi, reprodutores.

\section{NECROSIS OF THE FREE END OF THE PENIS AS COMPLICATION OF ACROPOSTITIS-PHIMOSIS IN BULL}

\begin{abstract}
Among the diseases that affect the external genitalia of bulls the acropostitephimosis deserves emphasi. In some cases can be diagnosed severe mucosal involvement of the internal leaflet preputial which can cripple surgery. Among the complications, when opting for surgery, is the compromising the free end of the penis that has not been appointed as common sequela. This paper aims to describe the clinical and histopathological findings of an unusual case of stenosis and fibrosis of
\end{abstract}


the inner preputial leaflet with resultant necrosis of the free end of the penis, as complications after surgery acropostite - phimosis. During the procedure, fragments of damaged areas for histopathology analysis were collected. Unable to surgical correction of the prepuce phimosis, amputation of penile segment was required. The procedure was backed by histopathologic findings that confirmed the gravity of the case. The histopathological report revealed glans with extensive surface and focally necrotic areas among other findings.

KEYWORDS: Breeder, cattle, coeundi impotence, external genitalia.

\section{INTRODUÇÃO}

A acropostite-fimose é uma alteração reprodutiva de destaque dentre aquelas que afetam a genitália externa do touro zebuíno, tendo relevância cirúrgica importante, principalmente em touros de alto valor genético (RABELO et al., 2015). Caracteriza-se por determinar inflamação na extremidade do prepúcio estando geralmente associada ao estreitamento do óstio prepucial, dificultando ou impedindo a exposição peniana (MARQUES et al., 1988; RABELO et al., 2006; NASCIMENTO \& SANTOS, 2011). As particularidades morfológicas e anatômicas podem predispor à ocorrência da enfermidade (ASHDOWN, 2006). Acrescente-se a este fato, pastagens mal manejadas com alturas elevadas, ervas daninhas, plantas espinhosas e/ou lenhosas, ectoparasitas e traumas mecânicos provocados por bicadas de aves de rapina e domésticas, atuando como importantes fatores de risco (DESROCHERS et al., 1995; RABELO et al., 2008, CAMPOS et al., 2015).

Os sinais clínicos mais evidentes incluem a dificuldade ou impossibilidade de efetuar a cópula, edema, necrose da mucosa prolapsada, miíases, hemorragia, abscesso, hipertermia local e disúria (RABELO \& SILVA, 2011).

O tratamento conservativo e ou cirúrgico é conduzido mediante a avaliação do quadro clínico do paciente, valor zootécnico do animal, infraestrutura da propriedade e mão-de-obra para condução do pós-operatório. Em muitas situações, em virtude do insucesso do tratamento conservativo, a opção pela cirurgia mostra-se como alternativa mais eficaz (RABELO et al., 2006).

Vários procedimentos cirúrgicos são descritos na literatura, porém os resultados são muito variáveis (RABELO \& SILVA, 2011). Todavia, independente da técnica preconizada, alguns aspectos previamente à intervenção mostram-se importantes e devem ser criteriosamente analisados pelo cirurgião. Em alguns casos, pode-se diagnosticar em touros portadores de acropostite-fimose, por ocasião do exame físico, severo comprometimento da mucosa do folheto prepucial interno, com ulcerações e fibrose extensa o que, independente do valor intrínseco do touro, inviabiliza a realização da cirurgia. Nestes casos, quando se opta pela intervenção cirúrgica, mesmo diante dessas complicações, o risco do animal apresentar exposição parcial permanente da parte livre do pênis mostra-se evidente, predispondo a traumas e consequente parafimose, inviabilizando o touro para reprodução. A fimose do óstio prepucial também é caracterizada como intercorrência importante e comum após a realização da cirurgia para o tratamento da acropostitefimose.

Dentre as várias complicações resultantes da acropostite em touros, o comprometimento da extremidade livre do pênis não tem sido apontado como sequela frequente (RABELO \& SILVA, 2011). Assim, o presente trabalho objetivou descrever os achados clínico-patológicos, bem como o tratamento cirúrgico de um caso incomum de necrose da extremidade livre do pênis secundária a acropostitefimose em touro. 


\section{MATERIAL E MÉTODOS}

Um touro Nelore, de cinco anos de idade, $900 \mathrm{~kg}$ de peso corporal e de alto valor zootécnico, apresentou histórico de acropostite com curso de seis meses e tratamento cirúrgico prévio por um veterinário. $O$ proprietário informou também que após o ato cirúrgico, além de não haver melhora da lesão, o bovino não conseguia mais expor o pênis pelo óstio prepucial (fimose) e apresentava dificuldade para urinar, sendo essa efetuada em forma de finos jatos de urina. Adicionalmente, foi informado a realização de tratamentos prévios com antibióticos sistêmicos e produtos tópicos a base de barbatimão (Stryphnodendron obovatum), todos sem resultados satisfatórios. Após prévio acordo com o proprietário, optou-se por avaliação minuciosa do aparelho genital externo com vistas à realização de nova cirurgia para possível resolução definitiva do problema.

No exame físico, realizado com auxílio de brete de contenção, não foram observadas alterações nos parâmetros vitais, como temperatura, frequências respiratória e cardíaca, movimentos ruminais e tempo de preenchimento capilar. Sequencialmente, realizou-se o exame específico da genitália externa. Notou-se severa estenose do óstio, com diâmetro de $1,5 \mathrm{~cm}$, impedindo a inspeção e avaliação da integridade da mucosa do folheto prepucial interno (Fig. 1).

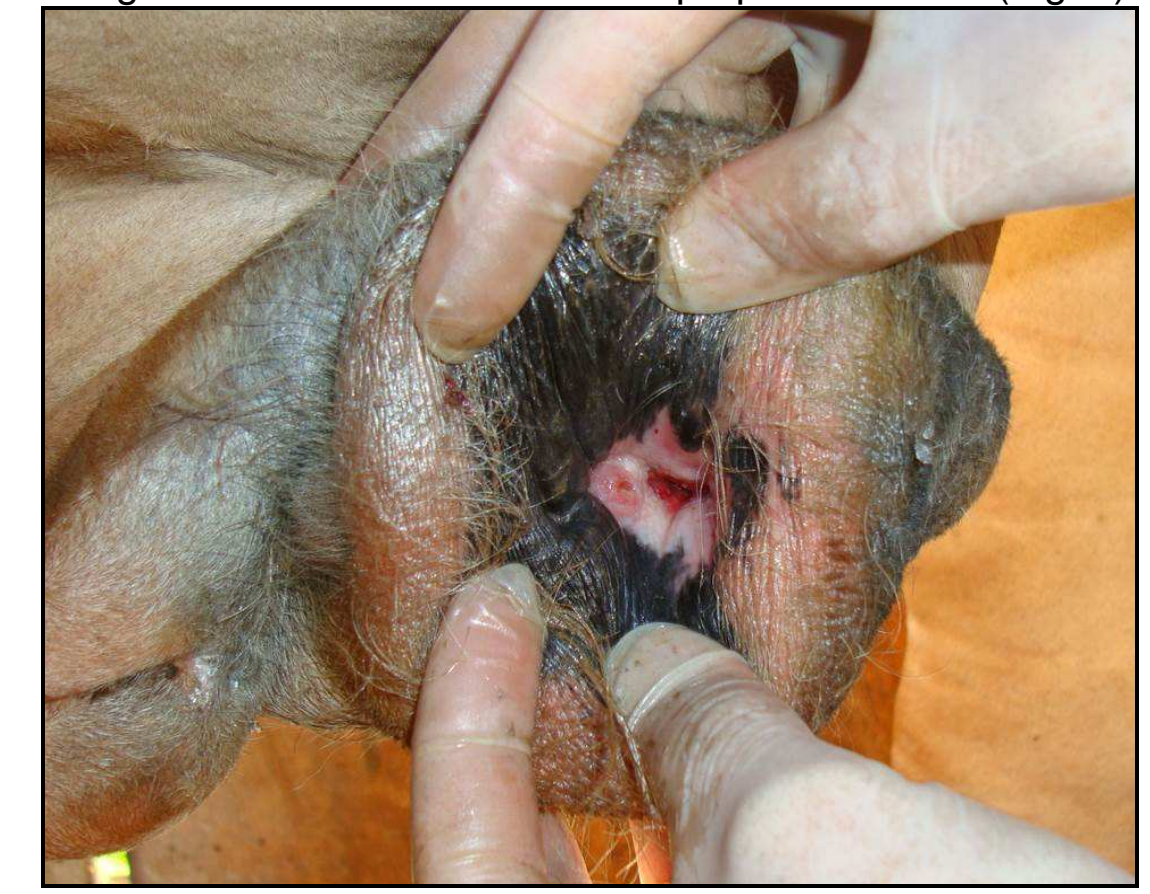

FIGURA 1. Bovino, prepúcio. Há estenose acentuada do óstio prepucial impossibilitando a avaliação do folheto prepucial interno. Fonte: Rabelo, 2014.

Diante do quadro acima descrito, a intervenção cirúrgica foi considerada a única opção de tratamento passível de ser realizada, caso o proprietário não optasse pelo descarte do animal. Entretanto, mediante a impossibilidade de mensurar a integridade da mucosa do folheto prepucial interno, argumentou-se com o proprietário dos potenciais riscos de insucesso, tanto durante o trans e pósoperatório, caso a opção pela cirurgia fosse considerada. Como se tratava de animal de alto valor zootécnico, o proprietário assumiu os riscos e optou pela realização da cirurgia.

Assim, o touro foi deixado em jejum completo de 24 horas e, posteriormente, submetido à sedação com cloridrato de xilazina a 2\% (Xilazin, 
Syntec do Brasil Ltda., Brasil) por via intravenosa na dosagem de $0,05 \mathrm{mg} / \mathrm{kg}$. O animal foi contido em decúbito lateral direito sendo preconizado o uso de almofadas de proteção visando prevenção de neurites e miosites. Para contenção dos membros locomotores empregou-se abraçadeiras em couro, evitando o contato direto das cordas.

Após preparo do campo operatório e lavagem intra-prepucial com solução à base de iodopovidine, procedeu-se a anestesia local infiltrativa, utilizando cloridrato de lidocaína a $2 \%$ sem vaso constritor (Dorfin, Hertape Calier Saúde Animal S. A, Brasil) circundando todo o óstio prepucial, empregando um volume máximo de $50 \mathrm{ml}$. A técnica cirúrgica baseou-se na circuncisão e remoção da fibrose visando ampliação do óstio prepucial conforme metodologia preconizada por RABELO \& SILVA (2011).

Por ocasião do ato cirúrgico, após circuncisão da área lesionada, verificou-se, por meio de manobras manuais e instrumentais de divulsão, que o folheto prepucial interno apresentava-se espesso e fibrosado, comprometendo aproximadamente $17 \mathrm{~cm}$ da mucosa da estrutura. Utilizando tesoura tipo romba reta, promoveu-se a abertura da óstio prepucial e constatou-se que a fibrose apresentava-se intensa, estenosando consideravelmente toda extensão do folheto prepucial interno. Nesse momento, foi observado que a extremidade da parte livre do pênis estava tumefeita e acentuadamente hiperêmica, indicando lesão isquêmica pela compressão do óstio prepucial fibrosado (Fig. 2). O óstio uretral também estava severamente estenosado.

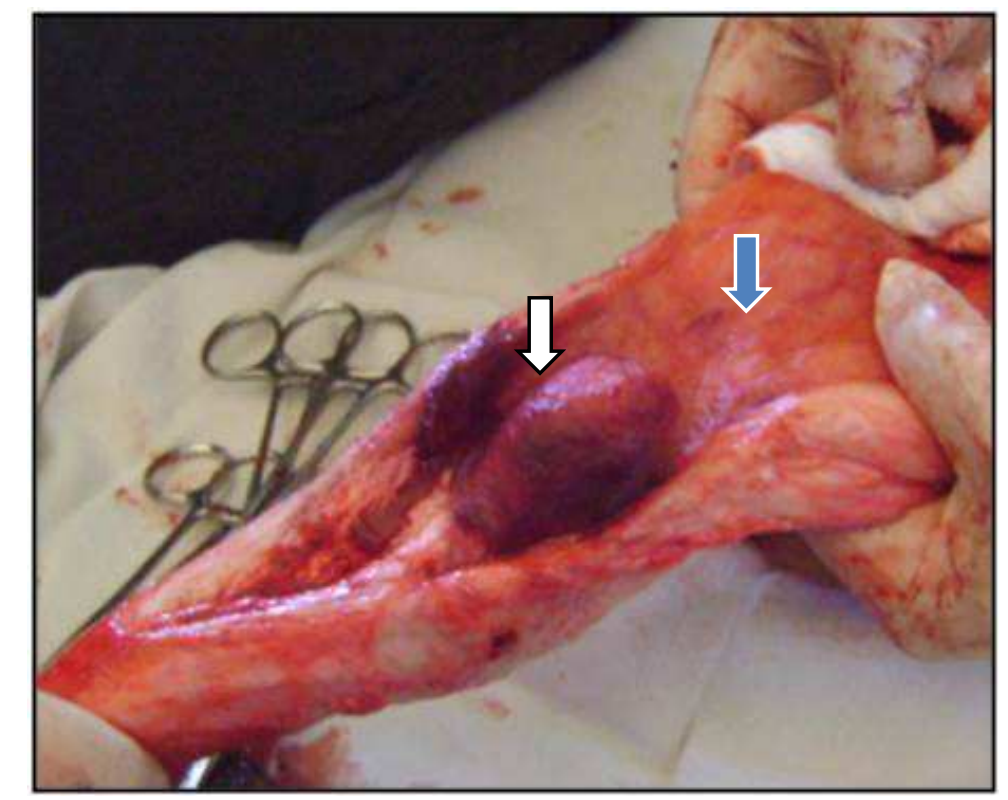

FIGURA 2. Bovino, pênis e prepúcio. Transoperatório demonstrou extensa estenose provocada pela fibrose na mucosa do folheto prepucial interno (seta azul). A seta branca indica o comprometimento isquêmico da extremidade livre do pênis. Fonte: Rabelo, 2014.

Diante dessa intercorrência transoperatória, optou-se pela amputação parcial do pênis, que foi realizada cranialmente à bolsa escrotal, sendo a incisão cutânea efetuada paralela à linha média e, após exposição do segmento do pênis a amputação, deixando um segmento peniano com comprimento médio de cinco $\mathrm{cm}$, e posterior fixação do coto peniano remanescente à pele. A sutura foi realizada em ENCICLOPÉDIA BIOSFERA, Centro Científico Conhecer - Goiânia, v.11 n.22; p.2299 2015 
padrão Wolff utilizando fio de poliamida, tendo o cuidado de fixar os pontos somente no tecido fibroso peniano, evitando a transfixação da uretra (RABELO \& SILVA, 2011). Finalizando o ato operatório, realizou-se a orquiectomia bilateral, empregando a técnica aberta de duas incisões laterais (SILVA et al., 2003).

Paralelamente, com o intuito de avaliar a severidade do comprometimento tecidual do segmento peniano afetado e sustentar a conduta cirúrgica estabelecida, foram coletados fragmentos da extremidade livre do pênis, sendo estes fixados e acondicionados em solução de formol neutro e tamponado a 10\% por 24 horas, para avaliação histopatológica.

Como medidas pós-operatórias estabeleceu-se antibioticoterapia a base de benzilpenicilina procaína (Penjet Max, Clarion Biociências Ltda., Brasil) na dose de $20.000 \mathrm{UI} / \mathrm{Kg}$ de peso corporal, de 24/24h por quatro aplicações. Prescreveu-se também anti-inflamatório não esteroidal a base de cetoprofeno (Ketojet $100 \mathrm{mg}$, Agener União saúde Animal, Brasil) na dosagem de $2 \mathrm{mg} / \mathrm{kg}$ de peso corporal por três dias seguidos e duchas com água sobre pressão no local e aplicação de sprays nas feridas cirúrgicas como auxiliares do processo de cicatrização. Estabeleceu-se a remoção dos pontos de pele decorridos 13 dias do ato cirúrgico, quando o bovino apresentava micção satisfatória e acentuada redução do edema no local.

O laudo histopatológico do fragmento peniano revelou glande com áreas focalmente extensas e superficiais de necrose com vasos neoformados e infiltrado inflamatório multifocal acentuado de neutrófilos, linfócitos e macrófagos. Notou-se também, em áreas teciduais mais profundas, focos isolados e discretos de necrose com o mesmo infiltrado inflamatório descrito anteriormente (Fig. 3).

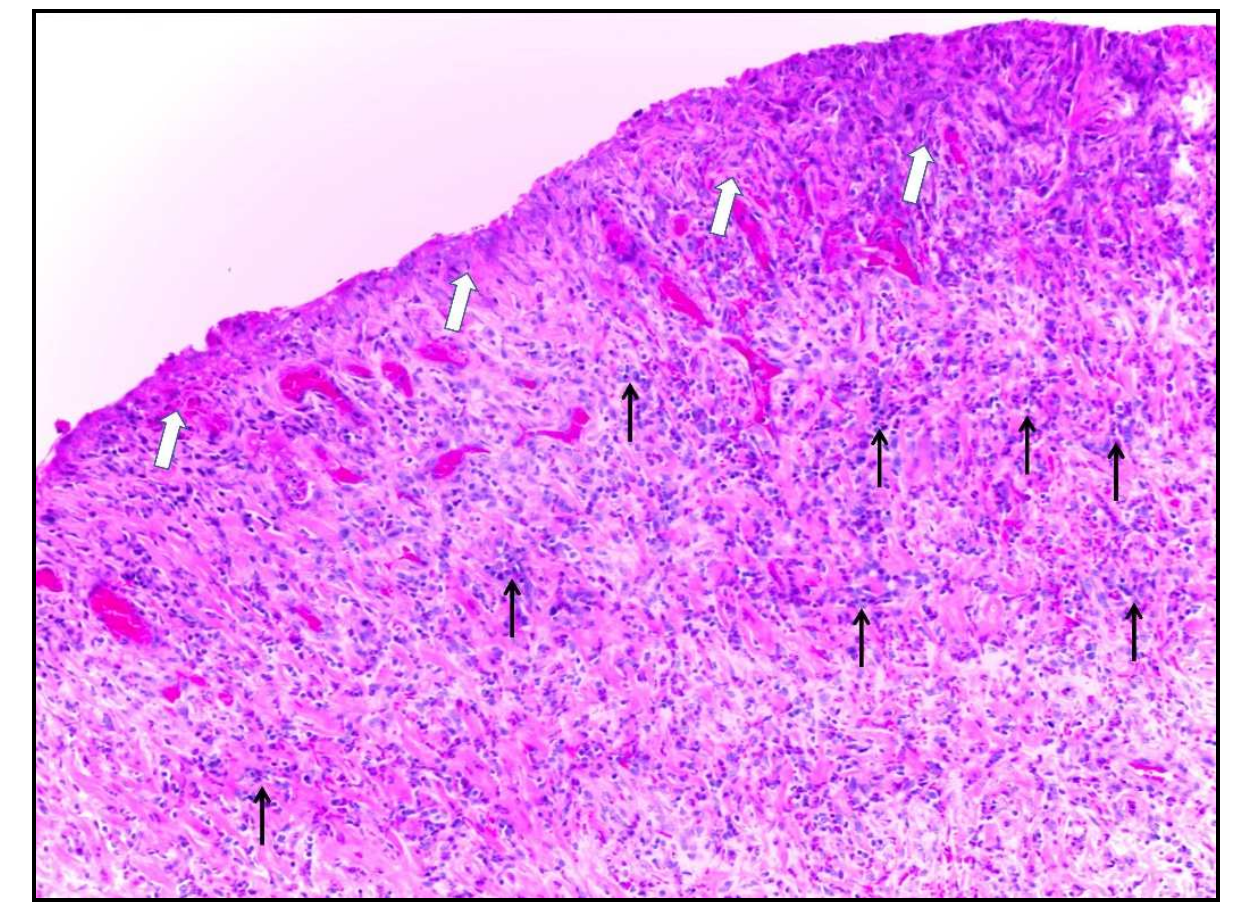

FIGURA 3 - Bovino, glande peniana. Observa-se área superficial focalmente extensa de necrose (setas brancas) com vasos neoformados adjacentes e infiltrado multifocal acentuado de linfócitos, neutrófilos e macrófagos (setas pretas). HE, Obj. 10X. Fonte: Rabelo, 2014. 


\section{RESULTADOS E DISCUSSÃO}

Embora a acropostite seja uma alteração reprodutiva comumente diagnosticada em touros zebuínos, principalmente pelo fato desses bovinos possuírem pênis e prepúcio penduloso e mais propício a traumatismos e lacerações (NASCIMENTO \& SANTOS, 2011, MENDONÇA et al., 2012), algumas complicações como necrose da extremidade livre do pênis não tem sido descritas na literatura especializada (FOSTER \& LADDS, 2007; RABELO \& SILVA, 2011).

A impossibilidade de realização de exame clínico específico, a fim de verificar a viabilidade da mucosa do folheto prepucial interno, em associação à fimose, dificultou a indicação do procedimento cirúrgico como opção de tratamento, bem como a determinação do prognóstico quanto à recuperação do paciente. Essa preocupação baseou-se no fato da importância de se avaliar clinicamente o real comprometimento da mucosa do folheto prepucial interno, principalmente quanto à presença de úlceras, aderências e fibrose extensa, que podem comprometer o sucesso do ato cirúrgico (RABELO et al., 2012). Assim, dependendo do segmento do folheto prepucial interno a ser removido, há risco eminente de o touro apresentar como intercorrência pós-operatória, exposição permanente da parte livre do pênis, culminando, posteriormente, na possibilidade do animal apresentar quadro clínico de parafimose. Esta preocupação encontra respaldo na literatura especializada, reforçando assim a importância e a necessidade da realização do exame clínico específico da genitália externa, empregando técnicas semiológicas ou mesmo métodos auxiliares, como a ultrassonografia, em casos de acropostite-fimose ou fimose, antes de optar pela intervenção cirúrgica (Rabelo et al., 2006; Rabelo \& Silva, 2011). Entretanto, devido à severa estenose do óstio prepucial, houve dificuldade de avaliar clinicamente esses detalhes e a dimensão desse comprometimento. Aliado a isso, a ausência de recursos auxiliares, como a ultrassonografia, mostrou-se como ponto de estrangulamento no referido atendimento. Entretanto, pelo fato do animal ser de alto valor genético e a remotas chances de recuperação do paciente, sendo a cirurgia a única opção apresentada, o proprietário manifestou seu posicionamento favorável quanto à realização do procedimento, mesmo diante dos reais riscos apresentados pela equipe técnica.

Provavelmente, o comprometimento severo da mucosa do folheto prepucial interno pela fibrose, promoveu a isquemia da extremidade livre do pênis e consequente necrose com comprometimento do óstio uretral, dificultando a micção. Esse fato foi apontado, no referido atendimento, como uma complicação que inviabilizou a técnica cirúrgica para correção da fimose prepucial e justificou a amputação do segmento peniano comprometido. Outros pesquisadores também fazem referência à técnica quando a comprometimento da extremidade peniana ou mesmo em alguns casos de urolitíases, parafimose ou intercorrência após o preparo de rufiões bovinos (RABELO et al., 2006; RABELO \& SILVA, 2011). Outro fator que, provavelmente, contribuiu com a necrose da glande peniana foi o represamento parcial de urina no folheto prepucial, uma vez que o bovino apresentava estenose acentuada do óstio prepucial. A avaliação histopatológica do segmento peniano comprometido evidenciou achados compatíveis com necrose superficial e balanite graves, dando respaldo à conduta técnica realizada no presente caso.

\section{CONCLUSÃO}

A fimose prepucial e fibrose do folheto prepucial interno, com consequente necrose da extremidade da parte livre do pênis como complicação cirúrgica da acropostite-fimose, mostrou ser um achado raro e de gravidade incontestável. A 
opção pela amputação do pênis apresentou-se como alternativa mais viável a ser executada, tendo em vista a gravidade do caso e o iminente risco de morte do paciente.

\section{REFERÊNCIAS}

ASHDOWN, R. R. Functional, developmental and clinical anatomy of the bovine penis and prepuce. CAB Reviews: Perspectives in Agriculture, Veterinary Science, Nutrition and Natural Resources, London, v.1, n.21, p.29-37, 2006. Disponível em: $<$ https://www.deepdyve.com/lp/centre-for-agricultural-bioscience-

international/functional-developmental-and-clinical-anatomy-of-the-bovine-penis-andxeiemyUdJi>. DOI: 10.1079/PAVSNNR20061021.

CAMPOS, L. G. M.; QUADROS, T. H. F.; CATALANO, F. A. R.; TUNIS JUNIOR, V. G.; FIGUEIREDO, J. C. Acrobustite em touro - Relato de caso. Anais do VI CONCCEPAR: Congresso Científico da Região Centro-Ocidental do Paraná / Faculdade Integrado de Campo Mourão. Campo Mourão, PR: Faculdade Integrado de Campo Mourão, 2015. Disponível em: <http://conccepar.grupointegrado.br/resumo/acrobustite-em-touro--relato-decaso/182>. ISSN 1983-7178.

DESROCHERS, A.; JEAN, G.; ANDERSON, D. E. Surgical management of injuries in bulls: 51 cases (1986-1994). Canadian Veterinary Journal, Ottawa, v.66, p.553556,1995. Disponível em: <http://www.ncbi.nlm.nih.gov/pmc/articles/PMC1687044/>.

FOSTER, R. A.; LADDS, P. W. Male genital system, In: JUBB, K. V. F.; KENNEDY, P. C.; PALMER, N. Pathology of domestic animals. St. Louis, MO: Elsevier Limited, p.590, 2007.

MARQUES, J. A.; MARQUES, L. C.; CANOLA, J. C.; CATTELAN, J. W. A acrobustite-fimose em touros - uma técnica cirúrgica de tratamento. Ciência Veterinária. v.2, n.1, p.2-3, 1988. ISSN 1517-6959.

MENDONÇA, A. C.; CARDOSO, J. R.; MOREIRA, P. C.; JUNQUEIRA, D. D.; MENDONÇA, F. P. X.; SILVA, M. S. B.; SILVA, D. B.; BRITO, L. A. B. Caracterização morfométrica do pênis e prepúcio de touros das raças nelore e gir. Bioscience Journal, Uberlândia, v.28, n.6, p.985-992, Nov./Dec. 2012. Disponível em: <http://www.seer.ufu.br/index.php/biosciencejournal/article/view/13327>. ISSN 1981-3163.

NASCIMENTO, E. F.; SANTOS, R. L. Patologia da reprodução dos animais domésticos. 3 ed. Rio de Janeiro: Gauanabara Koogan. p.153, 2011.

RABELO, R. E.; SILVA, L. A. F.; VIU, M. A. O. Acrobustite bovina: Revisão de literatura. Revista CFMV - Suplemento Técnico, Brasília. a.11, n.37, p. 29-36, 2006. ISSN 1517-6959.

RABELO, R. E.; SILVA, L. A. F.; BRITO, L. A. B. Epidemiological Aspects of surgical diseases of the genital tract in a population of 12,320 breeding bulls (1982-2007) in the state of Goias, Brazil. Ciência Animal Brasileira, v.9, n.3, p.705-713, 2008. Disponível em: <http://repositorio.bc.ufg.br/handle/ri/109>. ISSN 1089-6891. 
RABELO, R. E.; SILVA, O. C. Aspectos morfofucionais, clínicos e cirúrgicos do pênis, prepúcio e testículos de touros. Goiânia: Kelps, 2011.

RABELO, R. E.; VULCANI, V. A. S.; CARDOSO, L. D.; DUTRA, H. T.; HELRIGEL, P. A.; VINCENTIN, F. R. Aspectos anatômicos e sua relação com as enfermidades do prepúcio e pênis no touro. Revista Científica Eletrônica de Medicina Veterinária. a.9, $\quad$ n.18, 2012. Disponível em: < http://faef.revista.inf.br/imagens_arquivos/arquivos_destaque/atYEslptZwuCRI8_201 3-6-25-18-3-59.pdf>. ISSN 1679-7353.

RABELO, R. E.; SILVA, L. A. F.; VULCANI, V. A. S.; SANT'ANA, F. J. F.; ASSIS, B. M.; RABBERS, A. S. Enfermidades diagnosticadas na genitália externa de touros: Estudo retrospectivo (2007 - 2013), Cienc. anim. bras. v.16, n.1, p.133-143, jan./mar. $2015 . \quad$ Disponível em: < http://www.revistas.ufg.br/index.php/vet/article/view/30990/17880>. DOI: 10.1590/1089-68916i130990.

SILVA, L. A. F.; VIANA FILHO, P. R. L.; VERISSIMO, A. C. C. Efeito da estação do ano, da idade, do método de contenção e da técnica cirúrgica na recuperação clínica e no ganho de peso de bovinos submetidos a orquiectomia. Revista Brasileira de Saúde e Produção Animal, v.4, p.18-29, 2003. Disponível em: < http://revistas.ufba.br/index.php/rbspa/article/view/623/375.> ISSN 15199940. 